\title{
NUMERICAL SOLUTION OF POSITIVE SUM EXPONENTIAL EQUATIONS
}

\author{
C. MARTIN*, J. MILLER, AND KENT PEARCE
}

\begin{abstract}
A homotopy continuation method is applied to solve an inverse interpolation problem for representing data by a positive sum of decaying exponentials. The homotopy method transforms the interpolation problem to a problem of determining the roots of a given polynomial. The relative effectiveness of the continuation method is contrasted with several other rootfinding schemes.
\end{abstract}

\section{Transmitted by John Casti}

This communication describes the application of a continuation method to the solution of the exponential interpolation problem. Exponential interpolation problems are an important and frequently occurring class of problems which have a history of being numerically intractable. For applications and alternative methods of solution, the papers of Ruhe [6], Wiscome and Evans [9], and Evans et al. [1] should be consulted.

The problem of fitting a data set with a sum of exponentials is, in general, a problem of statistics. However, if the data set is small it is reasonable to ask if the data can be reproduced exactly, i.e., if the interpolation problem can be solved. The interpolation problem can, of course, be attacked by Prony's method, but we were interested in determining if the problem could be successfully mastered using the more direct approach of solving the set of nonlinear equations. In [2] we give a very detailed account of the continuation techniques. In this note we summarize the results of extensive numnerical simulation. The conclusion is that continuation methods are indeed competitive for problems of small dimensions under certain physically reasonable constraints.

The problem is formulated as follows: The expression

$$
y(t)=c_{1} e^{-\lambda_{1} t}+\cdots+c_{n} e^{-\lambda_{n} t}
$$

is sampled at $2 n$ equally spaced time intervals

$$
t=\Delta t, 2 \Delta t, \cdots, 2 n \Delta t,
$$

giving rise to $2 n$ equations in the $2 n$ unknowns $c_{1}, \cdots, c_{n}, \lambda_{1}, \cdots, \lambda_{n}$ :

*Supported in part by NSA Grant MDA904-85-4009 and NASA Grant NAG2-82. 


$$
\begin{aligned}
c_{1} e^{-\lambda_{1} \Delta t}+\cdots+c_{n} e^{-\lambda_{n} \Delta t} & =p_{1} \\
c_{1} e^{-2 \lambda_{1} \Delta t}+\cdots+c_{n} e^{-2 \lambda_{n} \Delta t} & =p_{2} \\
& \vdots \\
c_{1} e^{-2 n \lambda_{1} \Delta t}+\cdots+c_{n} e^{-2 n \lambda_{n} \Delta t} & =p_{2 n}
\end{aligned}
$$

If we set $x_{1}=e^{-\lambda_{1} \Delta t}, x_{2}=e^{-\lambda_{2} \Delta t}, \ldots, x_{n}=e^{-\lambda_{n} \Delta t}$, then (1) can be expressed as

$$
\begin{aligned}
c_{1} x_{1}+\cdots+c_{n} x_{n} & =p_{1} \\
c_{1} x_{1}^{2}+\cdots+c_{n} x_{n}^{2} & =p_{2} \\
& \vdots \\
c_{1} x_{1}^{2 n}+\cdots+c_{n} x_{n}^{2 n} & =p_{2 n}
\end{aligned}
$$

We assume the coefficient variables $c_{1}, \cdots, c_{n}$ are positive. This assumption is standard in the literature and, for many of the applications of exponential interpolation, is physically reasonable. Many of the techniques used to solve this problem, especially the least squares, become numen intractable without this assumption. The method we propose, continuation, likewise is unreliable if this assumption is not made.

We now consider $F: \mathbb{R}^{2 n} \rightarrow \mathbb{R}^{2 n}$ where $\mathbb{R}^{2 n}$ is $2 n$ dimensional euclidean space and the $j$ th coordinate function is given by

$$
f_{j}\left(c_{1}, \cdots, c_{n}, \lambda_{1}, \cdots, \lambda_{n}\right)=c_{1} x_{1}^{j}+\cdots+c_{n} x_{n}^{j}-p_{j}
$$

The system of polynomial equations (2) is to be solved by finding the zero of the mapping $F$ by a continuation method which we now describe. (The description of this method may be found in greater detail in Section 2 of Miller [3].)

The idea of this method is to use the mapping $F$ to lift curves of the form $(1-t) F(a)$, where $0 \leq t \leq 1$ and $a$ is in a bounded convex set $C$ containing the solution, to curves $x(t)=\left(x_{1}(t), \ldots, x_{2 n}(t)\right)$ which lie in $C$ and for which $x(0)=a$. Then, since $F(x(t))=(1-t) F(a)$, the zero of $F$ is clearly $x^{*}=x(1)$. This solution is found by considering $x(t)$ as a trajectory of the differential equation

$$
F^{\prime}(x(t)) \frac{d x}{d t}=F(a)
$$

with initial condition $x(0)=a$; the solution is then continued from $t=0$ to $t=1$.

In order for this method to work it is necessary that two conditions hold. First, $J F(x)$, the Jacobian of $F$ at $x$, must be nonzero at points of $C$. A calculation found in Martin, Miller, and Pearce [2] shows that $J F=0$ occurs only when $c_{1}=\cdots=c_{n}=x_{1}=\cdots=x_{n}=0$ or $x_{i}=x_{j}$. Inasmuch as the coordinates of the root of (1) must all be positive, $C$ can be taken to be a region in $\mathbb{R}^{2 n}$ which consists of positive coordinates and satisfies inequalities of the form $x_{i}<x_{j}$ for appropriate choices of $i$ and $j$. 
Secondly, for $a$ in $C, F(a)$ must fall in $S t(0)$, the star of 0 , which is defined as follows: Let $\partial C$ be the boundary of $C$, and $U$ the nonempty connected component of $\mathbb{R}^{2 n} \backslash F(\partial C)$ containing the origin. The point $y$ in $U$ is in $S t(0)$ provided the segment $(1-t) y$ lies in $U$ for all $0 \leq t \leq l$. Our experience was that these two conditions were met in all test problems we considered.

As noted above, to calculate the zero of $\mathrm{F}$ we solved

$$
F^{\prime}(x(t)) \frac{d x}{d t}=F(a)
$$

for the trajectory that passes through $a$, which can be done by making use of numerical methods. We used two methods in this paper.

The first method used was a packaged differential equation solver ODE described by Shampire and Gordon [7]. The second method used a predictor-corrector pathfollowing procedure to trace the covering path from a to the zero of $\mathrm{F}$. The details of this method will appear in Martin, Miller, and Pearce [2].

The homotopy methods used were compared against various methods of varying complexity, which were as follows: (1) both direct and modified (quasi-trust-region) forms of Newton's method, (2) a package nonlinear solver NS01A written by M. J. D. Powell [5], and (3) an IMSL package nonlinear solver DNEQNF explained in the IMSL Math/Library User's Manual [10], which was based on a MINPACK subroutine HBRD1 as found in Moré, Garbow, and Hillstrom [4].

The comparisons were carried out on standard sets of 1000 randomly generated problems for given $\mathrm{n}$ of 3 and 4. Newton's method was by far the weakest; NS01A was only moderately successful. It solved only $79 \%$ of the test problems for $n=3$ and $55 \%$ for $n=4$. DNEQNF did less well yet. It solved only $56 \%$ of the test problems with dimension $n=3$, and only $34 \%$ with dimension $n=4$.

On the other hand, ODE solved all the test problems in dimension $n=3$ and $99 \%$ of those in dimension $n=4$. We found, however, the program generally stagnated at dimensions $n$ between 6 and 8 .

The predictor-corrector scheme we used was very robust and very fast, and cheaper to use than ODE. It solved 93\% of the problems in dimension $n=3$ and $83 \%$ in dimension $n=4$. Failure invariably was due to coalescing of the $x_{j}$ components of the solution.

When more general problems for an unknown dimension less than 7 were considered, predictor-corrector techniques produce very reliable results. For problems with $n>7$, the program suffered difficulties, which suggested that a better predictor, such as a higher order Hermite extrapolator, might be required. [We used a third order polynomial predictor which was constructed from a form of (two-point) Hermite extrapolation.]

\section{REFERENCES}

[1] J. W. Evans, W. Gragg, and R. J. LeVegue, On least square exponential sum approximation with positive coefficients, Math Comp. 34(149):203-P.ll(Jan. 1980).

[2] C. Martin, J. Miller, and K. Pearce, Parameter identification by continual ${ }_{i}$, methods, Appl. Math. Comput., submitted for publication.

[3] J. Miller, Finding roots of equations in given neighborhoods, Appl. Math. Comput. 23(3):185-192 (Sept. 1987).

[4] J. Moré, B. Garbow, and K. Hillstrom, User Guide for MINPACK-l, Report ANL-80-74, Argonne National Labs., Argonne, Ill., 1980. 
[5] M. J. D. Powell, Technical Report AERE-R-5947, Harwell, England, 1968.

[6] A. Ruhe, Fitting empirical data by positive sums of exponentials, SIAM J. Sci.Statist. Comput. 1(1):481 498 (Dec. 1980).

[7] L. Shampire and M. Gordon, Computer Solution of Ordinary Differentiat Equations: The Initial Value Problem, Freeman, San Francisco, 1975.

[8] L. Watson, T. C. Biillups, and A. P. Morgan, Algorithms G52:HOMPACK: A suite of codes for globally convergent homotopy algorithms, ACM Trans. Math. Software 13(3):281 310 (1987).

[9] W. J. Wiscome and J. W. Evans, Exponential-sum fitting of radioactive transmission functions, J. Comput. Phys. 24:416-444 (1977).

[10] IMSL Math/Library, User's Manual 2, pp. 776 779, 1987.

Department of Mathematics, Texas Tech University, Lubbock, TX 79409

E-mail address: martin@math.ttu.edu

Department of Mathematics, Texas Tech University, Lubbock, TX 79409

E-mail address: miller@math.ttu.edu

Department of Mathematics, Texas Tech University, Lubbock, TX 79409

E-mail address: pearce@math.ttu.edu 